\title{
Non-classical heat conduction problem with nonlocal source
}

\author{
Mahdi Boukrouche and Domingo A Tarzia2*
}

"Correspondence:

DTarzia@austral.edu.ar

${ }^{2}$ Departamento de

Matemática-CONICET, FCE,

Universidad Austral, Paraguay 1950,

Rosario, S2000FZF, Argentina

Full list of author information is

available at the end of the article

\begin{abstract}
We consider the non-classical heat conduction equation, in the domain $D=\mathbb{R}^{n-1} \times \mathbb{R}^{+}$, for which the internal energy supply depends on an integral function in the time variable of the heat flux on the boundary $S=\partial D$, with homogeneous Dirichlet boundary condition and an initial condition. The problem is motivated by the modeling of temperature regulation in the medium. The solution to the problem is found using a Volterra integral equation of second kind in the time variable $t$ with a parameter in $\mathbb{R}^{n-1}$. The solution to this Volterra equation is the heat flux $(y, s) \mapsto$ $V(y, t)=u_{x}(0, y, t)$ on $S$, which is an additional unknown of the considered problem. We show that a unique local solution, which can be extended globally in time, exists. Finally a one-dimensional case is studied with some simplifications. We obtain the solution explicitly by using the Adomian method, and we derive its properties.
\end{abstract}

MSC: 35C15; 35K05; 35K20; 35K60; 45D05; 45E10; 80A20

Keywords: non-classical $n$-dimensional heat equation; nonlocal sources; Volterra integral equation; existence and uniqueness of solution; integral representation of solution; explicit solution in one-dimensional case and its properties

\section{Introduction}

Let us consider the domain $D$ and its boundary $S$ defined by

$$
\begin{aligned}
& D=\mathbb{R}^{n-1} \times \mathbb{R}^{+}=\left\{(x, y) \in \mathbb{R}^{n}: x=x_{1}>0, y=\left(x_{2}, \ldots, x_{n}\right) \in \mathbb{R}^{n-1}\right\}, \\
& S=\partial D=\mathbb{R}^{n-1} \times\{0\}=\left\{(x, y) \in \mathbb{R}^{n}: x=0, y \in \mathbb{R}^{n-1}\right\} .
\end{aligned}
$$

The aim of this paper is to study the following Problem 1.1 with a non-classical heat-flow feedback problem in the domain $D$ with nonlocal source, for which the internal energy supply depends on the integral $\int_{0}^{t} u_{x}(0, y, s) d s$ on the boundary $S$.

Problem 1.1 Find the temperature $u$ at $(x, y, t)$ such that it satisfies the following conditions:

$$
\begin{aligned}
& u_{t}-\Delta u=-F\left(\int_{0}^{t} u_{x}(0, y, s) d s\right), \quad x=x_{1}>0, y \in \mathbb{R}^{n-1}, t>0, \\
& u(0, y, t)=0, \quad y \in \mathbb{R}^{n-1}, t>0 \\
& u(x, y, 0)=h(x, y), \quad x>0, y \in \mathbb{R}^{n-1},
\end{aligned}
$$

(c) The Author(s) 2017. This article is distributed under the terms of the Creative Commons Attribution 4.0 International License (http://creativecommons.org/licenses/by/4.0/), which permits unrestricted use, distribution, and reproduction in any medium, provided you give appropriate credit to the original author(s) and the source, provide a link to the Creative Commons license, and indicate if changes were made. 
where $\Delta$ denotes the Laplacian in $\mathbb{R}^{n}$. This problem is motivated by the modeling of temperature regulation in an isotropic medium, with non-uniform and nonlocal sources that provide cooling or heating system; this could represent a feedback air-conditioning system in a macro scale installation. According to the properties of the function $F$ with respect to the heat flow, $V(y, s)=u_{x}(0, y, s)$ at the boundary $S$. For example, assuming that

$$
V \mathcal{F}(V)>0, \quad \forall V \neq 0, \mathcal{F}(0)=0,
$$

with

$$
\mathcal{F}(V(y, t))=F\left(\int_{0}^{t} V(y, s) d s\right)
$$

then, see $[1,2]$, the cooling source occurs when $V(y, t)>0$ and the heating source occurs when $V(y, t)<0$.

Some references on the subject are [3] where $\mathcal{F}(V)=F(V)$ and [4-7] where the following semi-one-dimension of this nonlinear problem was considered. The non-classical onedimensional heat equation in a slab with fixed or moving boundaries was studied in [811]. More references on the subject can be found in [12-16]. To our knowledge, it is the first time that the solution to a non-classical heat conduction of the type of Problem 1.1 is given. Other non-classical problems can be found in [17].

The goal of this paper is to obtain in Section 2 the existence and uniqueness of the global solution of the non-classical heat conduction Problem 1.1, which is given through a Volterra integral equation. In Section 3 we obtain the explicit solution of the onedimensional case of Problem 1.1, with some simplifications, which is obtained by using the Adomian method through a double induction principle.

We recall here the Green's function for the $n$-dimensional heat equation with homogeneous Dirichlet's boundary conditions, given the following expression $[18,19]$

$$
G_{1}(x, y, t ; \xi, \eta, \tau)=\frac{\exp \left[-\frac{\|y-\eta\|^{2}}{4(t-\tau)}\right]}{(2 \sqrt{\pi(t-\tau)})^{n-1}} G(x, t, \xi, \tau),
$$

where $G$ is the Green's function for the one-dimensional case given by

$$
G(x, t, \xi, \tau)=\frac{e^{-\frac{(x-\xi)^{2}}{4(t-\tau)}}-e^{-\frac{(x+\xi)^{2}}{4(t-\tau)}}}{2 \sqrt{\pi(t-\tau)}}, \quad t>\tau .
$$

\section{Existence results}

In this section, we give first in Theorem 2.1 the integral representation (2.1) of the solution of considered Problem 1.1, but it depends on the heat flow $V$ on the boundary $S$, which satisfies Volterra integral equation (2.3) with initial condition (2.4). Then we prove, in Theorem 2.3, under some assumptions on the data, that there exists a unique solution of Problem 1.1 locally in times which can be extended globally in times. 
Theorem 2.1 The integral representation of a solution of considered Problem 1.1 is given by the following expression:

$$
\begin{aligned}
u(x, y, t)= & u_{0}(x, y, t) \\
& -\int_{0}^{t} \frac{\operatorname{erf}\left(\frac{x}{2 \sqrt{t-\tau}}\right)}{(2 \sqrt{\pi(t-\tau)})^{n-1}}\left[\int_{\mathbb{R}^{n-1}} \exp \left[-\frac{\|y-\eta\|^{2}}{4(t-\tau)}\right] \mathcal{F}(V(\eta, \tau)) d \eta\right] d \tau,
\end{aligned}
$$

where

$$
\operatorname{erf}(\zeta)=\left(\frac{2}{\sqrt{\pi}} \int_{0}^{\zeta} e^{-X^{2}} d X\right)
$$

is the error function, with

$$
u_{0}(x, y, t)=\int_{D} G_{1}(x, y, t ; \xi, \eta, 0) h(\xi, \eta) d \xi d \eta
$$

and the heat flux $V(y, t)=u_{x}(0, y, t)$ on the surface $x=0$ satisfies the following Volterra integral equation:

$$
\begin{aligned}
V(y, t)= & V_{0}(y, t) \\
& -2 \int_{0}^{t} \frac{1}{(2 \sqrt{\pi(t-\tau)})^{n}}\left[\int_{\mathbb{R}^{n-1}} \exp \left[-\frac{\|y-\eta\|^{2}}{4(t-\tau)}\right] \mathcal{F}(V(\eta, \tau)) d \eta\right] d \tau
\end{aligned}
$$

in the variable $t>0$, with $y \in \mathbb{R}^{n-1}$ is a parameter and

$$
V_{0}(y, t)=\int_{D} G_{1, x}(0, y, t ; \xi, \eta, 0) h(\xi, \eta) d \xi d \eta
$$

where the function $(y, t) \mapsto \mathcal{F}(V(y, t))$ is defined by (1.4) for $y \in \mathbb{R}^{n-1}$ and $t>0$.

Proof As the boundary condition in Problem 1.1 is homogeneous, we have from [18]

$$
\begin{aligned}
u(x, y, t)= & \int_{D} G_{1}(x, y, t ; \xi, \eta, 0) h(\xi, \eta) d \xi d \eta \\
& +\int_{0}^{t} \int_{D} G_{1}(x, y, t ; \xi, \eta, \tau)[-\mathcal{F}(V(\eta, \tau))] d \xi d \eta d \tau
\end{aligned}
$$

and therefore

$$
\begin{aligned}
u_{x}(x, y, t)= & \int_{D} G_{1, x}(x, y, t ; \xi, \eta, 0) h(\xi, \eta) d \xi d \eta \\
& +\int_{0}^{t} \int_{D} G_{1, x}(x, y, t ; \xi, \eta, \tau)[-\mathcal{F}(V(\eta, \tau))] d \xi d \eta d \tau .
\end{aligned}
$$

From (1.5) (the definition of $G_{1}$ ) by derivation with respect to $x$, then taking $x=0$, we obtain

$$
\int_{D} G_{1, x}(0, y, t ; \xi, \eta, \tau) \mathcal{F}(V(\eta, \tau)) d \xi d \eta
$$




$$
\begin{aligned}
& =\int_{\mathbb{R}^{n-1}} \frac{\mathcal{F}(V(\eta, \tau)) e^{-\frac{\|y-\eta\|^{2}}{4(t-\tau)}}}{(t-\tau)^{\frac{n+2}{2}}(2 \sqrt{\pi})^{n}}\left(\int_{0}^{+\infty} \xi e^{-\frac{\xi^{2}}{4(t-\tau)}} d \xi\right) d \eta \\
& =\frac{2}{(2 \sqrt{\pi(t-\tau)})^{n}} \int_{\mathbb{R}^{n-1}} \mathcal{F}(V(\eta, \tau)) e^{-\frac{\|y-\eta\|^{2}}{4(t-\tau)}} d \eta,
\end{aligned}
$$

as

$$
\int_{0}^{+\infty} \xi e^{-\frac{\xi^{2}}{4(t-\tau)}} d \xi=2(t-\tau)
$$

Thus, taking $x=0$ in (2.6) with (2.7), we get (2.3).

Also by (1.5) we obtain

$$
\begin{aligned}
& \int_{D} G_{1}(x, y, t ; \xi, \eta, \tau) \mathcal{F}(V(\eta, \tau)) d \xi d \eta \\
& \quad=\frac{1}{(2 \sqrt{\pi(t-\tau)})^{n}} \int_{D} e^{\frac{-\|y-\eta\|^{2}}{4(t-\tau)}}\left[e^{-\frac{(x-\xi)^{2}}{4(t-\tau)}}-e^{-\frac{(x+\xi)^{2}}{4(t-\tau)}}\right] \mathcal{F}(V(\eta, \tau)) d \xi d \eta \\
& =\frac{1}{(2 \sqrt{\pi(t-\tau)})^{n}} \int_{\mathbb{R}^{+}}\left[e^{-\frac{(x-\xi)^{2}}{4(t-\tau)}}-e^{-\frac{(x+\xi)^{2}}{4(t-\tau)}}\right] d \xi \int_{\mathbb{R}^{n-1}} e^{\frac{-\|y-\eta\|^{2}}{4(t-\tau)}} \mathcal{F}(V(\eta, \tau)) d \eta
\end{aligned}
$$

and by using

$$
\begin{aligned}
\int_{0}^{+\infty} e^{\frac{-(x-\xi)^{2}}{4(t-\tau)}} d \xi & =2 \sqrt{t-\tau}\left(\int_{-\infty}^{0} e^{-X^{2}} d X+\int_{0}^{\frac{x}{2 \sqrt{t-\tau}}} e^{-X^{2}} d X\right) \\
& =\sqrt{\pi(t-\tau)}\left(1+\operatorname{erf}\left(\frac{x}{2 \sqrt{t-\tau}}\right)\right)
\end{aligned}
$$

and

$$
\begin{aligned}
\int_{0}^{+\infty} e^{\frac{-(x+\xi)^{2}}{4(t-\tau)}} d \xi & =2 \sqrt{t-\tau}\left(\int_{0}^{+\infty} e^{-X^{2}} d X-\int_{0}^{\frac{x}{2 \sqrt{t-\tau}}} e^{-X^{2}} d X\right) \\
& =\sqrt{\pi(t-\tau)}\left(1-\operatorname{erf}\left(\frac{x}{2 \sqrt{t-\tau}}\right)\right)
\end{aligned}
$$

we get

$$
\int_{D} G_{1}(x, y, t ; \xi, \eta, \tau) \mathcal{F}(V(\eta, \tau)) d \xi d \eta=\frac{\operatorname{erf}\left(\frac{x}{2 \sqrt{t-\tau}}\right)}{(2 \sqrt{\pi(t-\tau)})^{n-1}} \int_{\mathbb{R}^{n-1}} e^{-\frac{\|y-\eta\|^{2}}{4(t-\tau)}} \mathcal{F}(V(\eta, \tau)) d \eta
$$

Taking this formula in (2.5), we obtain (2.1).

To solve Volterra integral equation (2.3), we rewrite it in a suitable form.

Lemma 2.2 Volterra integral equation (2.3) can be rewritten in the following form:

$$
\begin{aligned}
V(y, t)= & \frac{1}{t(2 \sqrt{\pi t})^{n}} \int_{\mathbb{R}^{+}} \xi e^{-\frac{\xi^{2}}{4 t}}\left(\int_{\mathbb{R}^{n-1}} e^{-\frac{\|y-\eta\|^{2}}{4 t}} h(\xi, \eta) d \eta\right) d \xi \\
& -\frac{2}{(2 \sqrt{\pi})^{n}} \int_{0}^{t} \frac{1}{(t-\tau)^{n / 2}} \int_{\mathbb{R}^{n-1}} \mathcal{F}(V(\eta, \tau)) e^{-\frac{\|y-\eta\|^{2}}{4(t-\tau)}} d \eta d \tau
\end{aligned}
$$


Proof Using the derivative, with respect to $x$, of (1.5), then taking $x=0$ and $\tau=0$, then taking the new expression of $V_{0}(y, t)$ in Volterra integral equation (2.3), we obtain (2.8).

Theorem 2.3 Assume that $h \in \mathcal{C}(D), F \in \mathcal{C}(\mathbb{R})$ and locally Lipschitz in $\mathbb{R}$, then there exists a unique solution of Problem 1.1 locally in times which can be extended globally in times.

Proof We know from Theorem 2.1 that, to prove the existence and uniqueness of the solution (2.1) of Problem 1.1, it is enough to solve Volterra integral equation (2.8). So we rewrite it again as follows:

$$
V(y, t)=f(y, t)+\int_{0}^{t} g(y, \tau, V(y, \tau)) d \tau
$$

with

$$
f(y, t)=\frac{1}{t(2 \sqrt{\pi t})^{n}} \int_{\mathbb{R}^{+}} \xi e^{-\frac{\xi^{2}}{4 t}}\left(\int_{\mathbb{R}^{n-1}} e^{-\frac{\|y-\eta\|^{2}}{4 t}} h(\xi, \eta) d \eta\right) d \xi
$$

and

$$
g(t, \tau, y, V(y, \tau))=-\frac{2(t-\tau)^{-n / 2}}{(2 \sqrt{\pi})^{n}} \int_{\mathbb{R}^{n-1}} \mathcal{F}(V(\eta, \tau)) e^{-\frac{\|y-\eta\|^{2}}{4(t-\tau)}} d \eta .
$$

We have to check conditions $\mathrm{H} 1$ to $\mathrm{H} 4$ in Theorem 1.1 page 87, and $\mathrm{H} 5$ and $\mathrm{H} 6$ in Theorem 1.2 page 91 in [20].

- The function $f$ is defined and continuous for all $(y, t) \in \mathbb{R}^{n-1} \times \mathbb{R}^{+}$, so H1 holds.

- The function $g$ is measurable in $(t, \tau, y, x)$ for $0 \leq \tau \leq t<+\infty, x \in \mathbb{R}, y \in \mathbb{R}^{n-1}$, and continuous in $x$ for all $(y, t, \tau) \in \mathbb{R}^{n-1} \times \mathbb{R}^{+} \times \mathbb{R}^{+}, g(y, t, \tau, x)=0$ if $\tau>t$, so here we need the continuity of

$$
V(\eta, \tau) \mapsto \mathcal{F}(V(\eta, \tau))=F\left(\int_{0}^{\tau} V(\eta, s) d s\right),
$$

which follows from the hypothesis that $F \in \mathcal{C}(\mathbb{R})$. So H2 holds.

- For all $k>0$ and all bounded sets $B$ in $\mathbb{R}$, we have

$$
\begin{aligned}
|g(y, t, \tau, X)| & \leq \frac{2}{(2 \sqrt{\pi})^{n}} \sup _{X \in B}|\mathcal{F}(X)|(t-\tau)^{-n / 2} \int_{\mathbb{R}^{n-1}} e^{-\|y-\eta\|^{\frac{2}{4(t-\tau)}}} d \eta \\
& \leq \frac{2}{(2 \sqrt{\pi})^{n}} \sup _{X \in B}|\mathcal{F}(X)|(t-\tau)^{-n / 2}(2 \sqrt{\pi(t-\tau)})^{n-1} \\
& =\frac{1}{\sqrt{\pi}} \sup _{X \in B}|\mathcal{F}(X)| \frac{1}{\sqrt{(t-\tau)}},
\end{aligned}
$$

thus there exists a measurable function $m$ given by

$$
m(t, \tau)=\frac{1}{\sqrt{\pi}} \sup _{X \in B}|\mathcal{F}(X)| \frac{1}{\sqrt{(t-\tau)}}
$$

such that

$$
|g(y, t, \tau, X)| \leq m(t, \tau) \quad \forall 0 \leq \tau \leq t \leq k, X \in B
$$


and it satisfies

$$
\begin{aligned}
\sup _{t \in[0, K]} \int_{0}^{t} m(t, \tau) d \tau & =\frac{1}{\sqrt{\pi}} \sup _{X \in B}|\mathcal{F}(X)| \sup _{t \in[0, k]} \int_{0}^{t} \frac{1}{\sqrt{t-\tau}} d \tau \\
& =\frac{1}{\pi} \sup _{X \in B}|\mathcal{F}(X)| \sup _{t \in[0, k]}\left(-\left.2 \sqrt{(t-\tau)}\right|_{0} ^{t}\right) \\
& =\frac{1}{\pi} \sup _{X \in B}|\mathcal{F}(X)| \sup _{t \in[0, k]} \sqrt{t} \leq \frac{2 \sqrt{k}}{\pi} \sup _{X \in B}|\mathcal{F}(X)|<\infty,
\end{aligned}
$$

so $\mathrm{H} 3$ holds.

- Moreover, we have also

$$
\begin{aligned}
\lim _{t \rightarrow 0^{+}} \int_{0}^{t} m(t, \tau) d \tau & =\frac{1}{\sqrt{\pi}} \sup _{X \in B}|\mathcal{F}(X)| \lim _{t \rightarrow 0^{+}} \int_{0}^{t} \frac{d \tau}{\sqrt{t-\tau}} \\
& =\frac{1}{\sqrt{\pi}} \sup _{X \in B}|\mathcal{F}(X)| \lim _{t \rightarrow 0^{+}}(2 \sqrt{t})=0,
\end{aligned}
$$

and

$$
\lim _{t \rightarrow 0^{+}} \int_{T}^{T+t} m(t, \tau) d \tau=\frac{1}{\sqrt{\pi}} \sup _{X \in B}|\mathcal{F}(X)| \lim _{t \rightarrow 0^{+}}(2 \sqrt{t})=0
$$

- For each compact subinterval $J$ of $\mathbb{R}^{+}$, each bounded set $B$ in $\mathbb{R}^{n-1}$, and each $t_{0} \in \mathbb{R}^{+}$, we set

$$
\begin{aligned}
& \mathcal{A}(t, y, V(\eta))=\left|g(t, \tau ; y, V(\eta, \tau))-g\left(t_{0}, \tau ; y, V(\eta, \tau)\right)\right|, \\
& \mathcal{A}(t, y, V(\eta))=\frac{2}{(2 \sqrt{\pi})^{n}} \int_{J}\left|\int_{\mathbb{R}^{n-1}} e^{-\frac{\|y-\eta\|^{2}}{4(t-\tau)}} \frac{\mathcal{F}(V(\eta, \tau))}{(t-\tau)^{-n / 2}}-e^{-\frac{\|y-\eta\|^{2}}{4\left(t_{0}-\tau\right)}} \frac{\mathcal{F}(V(\eta, \tau))}{\left(t_{0}-\tau\right)^{-n / 2}} d \eta\right| d \tau
\end{aligned}
$$

as the function $\tau \mapsto V(\eta, \tau)$ is continuous, then

$$
\tau \mapsto \int_{0}^{\tau} V(\eta, s) d s
$$

is $\mathcal{C}^{1}(\mathbb{R})$ and is in the compact $B \subset \mathbb{R}$ for all $\eta \in \mathbb{R}^{n-1}$, so by the continuity of $\mathcal{F}$ we get $\mathcal{F}(V(\eta, \tau)) \subset \mathcal{F}(B)$. That is, there exists $M>0$ such that $|\mathcal{F}(V(\eta, \tau))| \leq M$ for all $(\eta, \tau) \in \mathbb{R}^{n-1} \times \mathbb{R}^{+}$. So

$$
\begin{aligned}
& \sup _{V(\eta) \in \mathcal{C}(J, B)} \mathcal{A}(t, y, V(\eta)) \\
& \quad \leq \frac{2 M}{(2 \sqrt{\pi})^{n}} \sup _{V(\eta) \in \mathcal{C}(J, B)}\left|\int_{\mathbb{R}^{n-1}} \frac{e^{-\frac{\|y-\eta\|^{2}}{4(t-\tau)}}}{\sqrt{(t-\tau)^{n}}} d \eta-\int_{\mathbb{R}^{n-1}} \frac{e^{-\frac{\|y-\eta\|^{2}}{4\left(t_{0}-\tau\right)}}}{\sqrt{\left(t_{0}-\tau\right)^{n}}} d \eta\right| .
\end{aligned}
$$

Using that

$$
\int_{\mathbb{R}^{n-1}} \exp \left[-\frac{\|y-\eta\|^{2}}{4(t-\tau)}\right] d \eta=(2 \sqrt{\pi(t-\tau)})^{n-1}
$$


we obtain

$$
\sup _{V(\eta) \in \mathcal{C}(J, B)} \mathcal{A}(t, y, V(\eta)) \leq \frac{2 M}{(2 \sqrt{\pi})^{n}} \sup _{V(\eta) \in \mathcal{C}(J, B)}\left|\frac{(2 \sqrt{\pi(t-\tau)})^{n-1}}{(\sqrt{t-\tau})^{n}}-\frac{\left(2 \sqrt{\pi\left(t_{0}-\tau\right)}\right)^{n-1}}{\left(\sqrt{t_{0}-\tau}\right)^{n}}\right|
$$

thus

$$
\sup _{V(\eta) \in \mathcal{C}(J, B)} \mathcal{A}(t, y, V(\eta)) \leq \frac{M}{\sqrt{\pi}} \sup _{V(\eta) \in \mathcal{C}(J, B)}\left|\frac{\sqrt{t_{0}-\tau}-\sqrt{t-\tau}}{\sqrt{(t-\tau)\left(t_{0}-\tau\right)}}\right| .
$$

Thus we deduce that

$$
\lim _{t \rightarrow t_{0}} \int_{J} \sup _{V(\eta) \in \mathcal{C}(J, B)} \mathcal{A}(t, y, V(\eta)) d \tau=0
$$

So H4 holds.

- For all compact $I \subset \mathbb{R}^{+}$, for all functions $\psi \in \mathcal{C}\left(I, \mathbb{R}^{n}\right)$, and all $t_{0}>0$,

$$
\begin{aligned}
& \left|g(t, \tau ; \psi(\tau))-g\left(t_{0}, \tau, \psi(\tau)\right)\right| \\
& \quad=\frac{2}{(2 \sqrt{\pi})^{n}}\left|\int_{\mathbb{R}^{n-1}} \mathcal{F}(\psi(\tau))\left(\frac{e^{-\frac{\|y-\eta\|^{2}}{4(t-\tau)}}}{(t-\tau)^{n / 2}}-\frac{e^{-\frac{\|y-\eta\|^{2}}{4\left(t_{0}-\tau\right)}}}{\left(t_{0}-\tau\right)^{n / 2}}\right) d \tau\right|
\end{aligned}
$$

as $\mathcal{F} \in \mathcal{C}(\mathbb{R})$ and $\psi \in \mathcal{C}\left(I, \mathbb{R}^{n}\right)$, then there exists a constant $M>0$ such that $|\mathcal{F}(\psi(\tau))| \leq M$ for all $\tau \in I$. Then we obtain, as for $\mathrm{H} 4$, that

$$
\lim _{t \rightarrow t_{0}} \int_{I}\left|g(t, \tau ; \psi(\tau))-g\left(t_{0}, \tau, \psi(\tau)\right)\right| d \tau=0
$$

So H5 holds.

- Now, for each constant $K>0$ and each bounded set $B \subset \mathbb{R}^{n-1}$, there exists a measurable function $\varphi$ such that

$$
|g(y, t, \tau, x)-g(y, t, \tau, X)| \leq \varphi(t, \tau)|x-X|
$$

whenever $0 \leq \tau \leq t \leq K$ and both $x$ and $X$ are in $B$. Indeed, as $F$ is assumed to be a locally Lipschitz function in $\mathbb{R}$, there exists a constant $L>0$ such that

$$
|\mathcal{F}(x)-\mathcal{F}(X)| \leq L(\tau)|x-X| \quad \forall(x, X) \in B^{2}
$$

with $L(\tau)=L \tau$. Then we have

$$
\begin{aligned}
|g(y, t, \tau, x)-g(y, t, \tau, X)| & =\frac{2}{(2 \sqrt{\pi})^{n}}\left|\int_{\mathbb{R}^{n-1}}(t-\tau)^{-n / 2} e^{-\frac{\|y-\eta\|^{2}}{4(t-\tau)}}(\mathcal{F}(x)-\mathcal{F}(X)) d \eta\right| \\
& \leq \frac{2}{(2 \sqrt{\pi})^{n}}\left(\int_{\mathbb{R}^{n-1}} e^{-\frac{\|y-\eta\|^{2}}{4(t-\tau)}} d \eta\right)(t-\tau)^{-n / 2} L \tau|x-X| \\
& \leq \frac{L \tau}{\sqrt{\pi(t-\tau)}}|x-X|,
\end{aligned}
$$


then $\varphi(t, \tau)=\frac{L \tau}{\sqrt{\pi(t-\tau)}}$. We have also for each $t \in[0, k]$ the function $\varphi \in L^{1}(0, t)$ as a function of $\tau$ and

$$
\begin{aligned}
\int_{t}^{t+l} \varphi(t+l, \tau) \tau d \tau & =\frac{L}{\sqrt{\pi}} \int_{t}^{t+l} \frac{\tau d \tau}{\sqrt{t+l-\tau}}=\frac{L}{\sqrt{\pi}} \int_{l}^{0}\left(u^{2}-t-l\right) d u \\
& =\frac{L l}{\sqrt{\pi}}\left(l+t-\frac{1}{3}\right) \rightarrow 0 \quad \text { with } l \rightarrow 0,
\end{aligned}
$$

where $u=\sqrt{t+l-\tau}$

So H6 holds. All the conditions H1 to H6 are satisfied with (2.14) and (2.15).

Thus from [20] (Theorem 1.1 p.87, Theorem 1.2 p.91 and Theorem 2.3 p.97) there exists a unique local times solution of Volterra integral equation (2.3) which can be extended globally in times. Then the proof of this theorem is complete.

\section{The one-dimensional case of Problem 1.1}

Let us consider now the one-dimensional case of Problem 1.1 for the temperature defined by

Problem 3.1 Find the temperature $u$ at $(x, t)$ such that it satisfies the following conditions:

$$
\begin{aligned}
& u_{t}-u_{x x}=-F\left(\int_{0}^{t} u_{x}(0, s) d s\right), \quad x>0, t>0, \\
& u(0, t)=0, \quad t>0 \\
& u(x, 0)=h(x), \quad x>0 .
\end{aligned}
$$

Taking into account that

$$
\int_{0}^{t} G(x, t, \xi, \tau) d \xi=\operatorname{erf}\left(\frac{x}{2 \sqrt{t-\tau}}\right)
$$

thus the solution of Problem 3.1 is given by

$$
u(x, t)=u_{0}(x, t)-\int_{0}^{t} \operatorname{erf}\left(\frac{x}{2 \sqrt{t-\tau}}\right) F\left(\int_{0}^{\tau} W(\sigma) d \sigma\right) d \tau
$$

with

$$
u_{0}(x, t)=\int_{0}^{t} G(x, t, \xi, 0) h(\xi) d \xi
$$

and $W(t)=u_{x}(0, t)$ is the solution of the following Volterra integral equation:

$$
W(t)=V_{0}(t)-\int_{0}^{t} \frac{F\left(\int_{0}^{\tau} W(\sigma) d \sigma\right)}{\sqrt{\pi(t-\tau)}} d \tau
$$

where

$$
V_{0}(t)=\frac{1}{2 \sqrt{\pi} t^{3 / 2}} \int_{0}^{+\infty} \xi e^{-\xi^{2} / 4 t} h(\xi) d \xi=\frac{2}{\sqrt{\pi t}} \int_{0}^{+\infty} \eta e^{-\eta^{2}} h(2 \sqrt{t} \eta) d \eta .
$$


For the particular case

$$
h(x)=h_{0}>0 \text { pour } x>0, \text { and } F(W)=\lambda W \text { for } \lambda \in \mathbb{R}
$$

we have

$$
u_{0}(t, x)=h_{0} \operatorname{erf}\left(\frac{x}{2 \sqrt{t}}\right)
$$

and integral equation (3.4) becomes

$$
W(t)=\frac{h_{0}}{\sqrt{\pi t}}-\lambda \int_{0}^{t} \frac{\int_{0}^{\tau} W(\sigma) d \sigma}{\sqrt{\pi(t-\tau)}} d \tau
$$

Lemma 3.1 Assume (3.6) holds. The solution of Problem 3.1 is given by

$$
u(x, t)=h_{0} \operatorname{erf}\left(\frac{x}{2 \sqrt{t}}\right)-\lambda \int_{0}^{t} \operatorname{erf}\left(\frac{x}{2 \sqrt{t-\tau}}\right) U(\tau) d \tau
$$

where $U$ is given by

$$
U(t)=\frac{h_{0}}{\sqrt{\pi}} \int_{0}^{t} \frac{g(\tau)}{\sqrt{t-\tau}} d \tau
$$

and $g$ is the solution of the Volterra integral equation

$$
g(t)=1-\frac{2 \lambda}{\sqrt{\pi}} \int_{0}^{t} g(\tau) \sqrt{t-\tau} d \tau .
$$

Moreover, the heat flux on $x=0$ is given by

$$
u_{x}(0, t)=U^{\prime}(t)=\frac{h_{0}}{\sqrt{\pi t}}-h_{0} \lambda \int_{0}^{t} g(\tau) d \tau, \quad t>0 .
$$

Proof We set

$$
U(t)=\int_{0}^{t} W(\tau) d \tau
$$

thus the function $U$ satisfies the following new Volterra integral equation:

$$
\begin{aligned}
U(t) & =2 h_{0} \sqrt{\frac{t}{\pi}}-\frac{\lambda}{\sqrt{\pi}} \int_{0}^{t} \int_{0}^{\tau} \frac{U(\sigma)}{\sqrt{\tau-\sigma}} d \sigma d \tau \\
& =2 h_{0} \sqrt{\frac{t}{\pi}}-\frac{2 \lambda}{\sqrt{\pi}} \int_{0}^{t} U(\tau) \sqrt{t-\tau} d \tau, \quad t>0
\end{aligned}
$$

by using the following equality:

$$
\int_{\sigma}^{t} \frac{d \tau}{\sqrt{\tau-\sigma}}=2 \sqrt{t-\sigma}, \quad 0<\sigma<t
$$


From [21], p.229, the solution $t \mapsto U(t)$ of integral equation (3.14) is given by (3.10), where $g$ is the solution of Volterra equation (3.11).

From (3.11) we obtain that

$$
\int_{0}^{t} \frac{g(\tau)}{\sqrt{t-\tau}} d \tau=2 \sqrt{t}-\lambda \sqrt{\pi} \int_{0}^{t} g(\tau) \sqrt{t-\tau} d \tau
$$

using the following equality:

$$
\begin{aligned}
\int_{\sigma}^{t} \frac{\sqrt{\tau-\sigma}}{\sqrt{t-\tau}} d \tau & =(t-\sigma) \int_{0}^{1} \frac{\sqrt{\xi}}{\sqrt{1-\xi}} d \xi=(t-\sigma) B\left(\frac{3}{2}, \frac{1}{2}\right) \\
& =(t-\sigma) \frac{\Gamma\left(\frac{3}{2}\right) \Gamma\left(\frac{1}{2}\right)}{\Gamma(2)}=\frac{\pi}{2}(t-\sigma),
\end{aligned}
$$

where $B$ and $\Gamma$ are the classical beta and gamma functions defined below.

Therefore, we have that

$$
U(t)=2 h_{0} \sqrt{\frac{t}{\pi}}-\lambda h_{0} \int_{0}^{t} g(\tau)(t-\tau) d \tau,
$$

and then the heat flux on $x=0$ is given by $u_{x}(0, t)=W(t)=U^{\prime}(t)$, that is, (3.12) holds.

We recall here the well-known beta and gamma functions defined respectively by

$$
\begin{aligned}
& B(x, y)=\int_{0}^{1} t^{x-1}(1-t)^{y-1} d t, \quad x>0, y>0, \\
& \Gamma(x)=\int_{0}^{+\infty} t^{x-1} e^{-t} d t, \quad x>0 .
\end{aligned}
$$

We will use in the next theorem the well-known relations

$$
\begin{aligned}
& B(x, y)=\frac{\Gamma(x) \Gamma(y)}{\Gamma(x+y)}, \quad \Gamma(x+1)=x \Gamma(x) \quad \forall x>0, \\
& \Gamma\left(\frac{1}{2}\right)=\sqrt{\pi}, \quad \Gamma(n+1)=n ! \quad \forall n \in \mathbb{N},
\end{aligned}
$$

and in particular the following one.

Lemma 3.2 For all integers $n \geq 1$, we have

$$
\Gamma\left(n+\frac{1}{2}\right)=\frac{(2 n-1) ! !}{2^{n}} \sqrt{\pi}
$$

and we use the definition

$$
(2 n-1) ! !=(2 n-1)(2 n-3)(2 n-5) \cdots 5 \cdot 3 \cdot 1
$$

for compactness expression. 
Proof For $n=1$, we get $\Gamma\left(\frac{3}{2}\right)=\frac{\sqrt{\pi}}{2}$, which is true. By induction we obtain that

$$
\begin{aligned}
\Gamma\left(n+1+\frac{1}{2}\right) & =\Gamma\left(\left(n+\frac{1}{2}\right)+1\right)=\left(n+\frac{1}{2}\right) \Gamma\left(n+\frac{1}{2}\right) \\
& =\left(n+\frac{1}{2}\right) \frac{(2 n-1) ! !}{2^{n}} \sqrt{\pi}=\frac{(2 n+1) ! !}{2^{n+1}} \sqrt{\pi}
\end{aligned}
$$

thus the lemma is true.

Corollary 3.3 For all integers $n \geq 0$, we have also

$$
\begin{aligned}
& \Gamma\left(3 n+5+\frac{1}{2}\right)=\frac{(6 n+9) ! !}{2^{3 n+5}} \sqrt{\pi} \\
& B\left(\frac{3}{2}, 3 n+4\right)=\frac{\Gamma\left(\frac{3}{2}\right) \Gamma(3(n+1)+1)}{\Gamma\left(3 n+5+\frac{1}{2}\right)}=\frac{(3(n+1)) ! 2^{3(n+1)+1}}{(6 n+9) ! !}, \\
& B\left(\frac{3}{2}, 3 n+\frac{5}{2}\right)=\frac{\Gamma\left(\frac{3}{2}\right) \Gamma\left(3 n+\frac{5}{2}\right)}{\Gamma(3(n+1)+1)}=\frac{\pi(6 n+3) ! !}{(3(n+1)) ! 2^{3(n+1)}}
\end{aligned}
$$

which will be useful in the next lemma.

First, we need some preliminary simple results in order to obtain the solution of integral equation (2.1).

$$
\begin{aligned}
& \int_{0}^{t} \sqrt{t-\tau} d \tau=\frac{2}{3} t^{3 / 2}, \quad \int_{0}^{t} \tau^{3 / 2} \sqrt{t-\tau} d \tau=\frac{\pi}{2^{4}} t^{3}, \\
& \int_{0}^{t} \tau^{3} \sqrt{t-\tau} d \tau=\frac{2^{4} 3 !}{9 ! !} t^{9 / 2}, \quad \int_{0}^{t} \tau^{9 / 2} \sqrt{t-\tau} d \tau=\frac{\pi 9 ! !}{2^{6} 6 !} t^{6} \\
& \int_{0}^{t} \tau^{6} \sqrt{t-\tau} d \tau=\frac{2^{7} 6 !}{15 ! !} t^{15 / 2}, \quad \int_{0}^{t} \tau^{15 / 2} \sqrt{t-\tau} d \tau=\frac{\pi 15 ! !}{2^{9} 9 !} t^{9},
\end{aligned}
$$

which can be generalized by the following ones.

Lemma 3.4 For all integers $n \geq 0$, we have

$$
\begin{aligned}
& \int_{0}^{t} \tau^{2 n+3} \sqrt{t-\tau} d \tau=\frac{2^{3 n+4}(3(n+1)) !}{(6 n+9) ! !} t^{3(2 n+3) / 2}, \\
& \int_{0}^{t} \tau^{\frac{3(2 n+1)}{2}} \sqrt{t-\tau} d \tau=\frac{\pi(6 n+3) ! !}{2^{3(n+1)(3(n+1)) !}} t^{3(n+1)}
\end{aligned}
$$

Proof Taking the change of variable $\tau=t \xi$ in (3.22) and using Corollary 3.3, we get

$$
\begin{aligned}
\int_{0}^{t} \tau^{2 n+3} \sqrt{t-\tau} d \tau & =t^{\frac{3(2 n+3)}{2}} \int_{0}^{1} \xi^{3 n+3}(1-\xi)^{\frac{1}{2}} d \xi \\
& =t^{\frac{3(2 n+3)}{2}} \int_{0}^{1} \xi^{(3 n+4)-1}(1-\xi)^{\frac{3}{2}-1} d \xi \\
& =t^{\frac{3(2 n+3)}{2}} B\left(\frac{3}{2}, 3 n+4\right)=\frac{(3(n+1)) ! 2^{3(n+1)+1}}{(6(n+9)) ! !}
\end{aligned}
$$


and

$$
\begin{aligned}
\int_{0}^{t} \tau^{\frac{3(2 n+1)}{2}} \sqrt{t-\tau} d \tau & =t^{\frac{3(2 n+1)}{2}} \int_{0}^{1} \xi^{\left(3 n+\frac{5}{2}\right)-1}(1-\xi)^{\frac{3}{2}-1} d \xi \\
& =t^{\frac{3(2 n+1)}{2}} B\left(\frac{3}{2}, 3 n+\frac{5}{2}\right)=\frac{\pi(6 n+3) ! !}{(3(n+1)) ! 2^{3(n+1)}} t^{3(n+1)}
\end{aligned}
$$

thus (3.22)-(3.23) hold.

Now, we will obtain the explicit solution of the integral equation

$$
y(t)=1-\frac{2 \lambda}{\sqrt{\pi}} \int_{0}^{t} y(\tau) \sqrt{t-\tau} d \tau, \quad t>0,
$$

by using the Adomian decomposition method [22-24] through a series expansion.

Theorem 3.5 The solution of integral equation (3.24) is given by the following expression:

$$
y(t)=I(t)-\sqrt{\frac{2}{\pi}} J(t), \quad t>0
$$

with

$$
I(t)=\sum_{n=0}^{+\infty} \frac{\left(\lambda^{2 / 3} t\right)^{3 n}}{(3 n) !}
$$

and

$$
J(t)=\sum_{n=0}^{+\infty} \frac{\left(\lambda^{2 / 3} t\right)^{\frac{3(2 n+1)}{2}}}{(3(2 n+1)) ! !}
$$

are series with infinite radii of convergence.

Proof Following the idea of [25-33], we propose, for the solution of integral equation (3.24), the series of expansion functions given by

$$
y(t)=\sum_{n=0}^{+\infty} y_{n}(t)
$$

and we obtain the following recurrence expressions:

$$
y_{0}(t)=1, \quad y_{n}(t)=-\frac{2 \lambda}{\sqrt{\pi}} \int_{0}^{t} y_{n-1}(\tau) \sqrt{t-\tau} d \tau, \quad \forall n \geq 1
$$

Then we get

$$
\begin{aligned}
& y_{1}(t)=-\frac{2 \lambda}{\sqrt{\pi}} \int_{0}^{t} \sqrt{t-\tau} d \tau=-\frac{4 \lambda}{3 \sqrt{\pi}} t^{3 / 2}=-\sqrt{\frac{2}{\pi}} \frac{\left(2 \lambda^{2 / 3} t\right)^{3 / 2}}{3 ! !}, \\
& y_{2}(t)=-\frac{2 \lambda}{\sqrt{\pi}} \int_{0}^{t}\left(-\frac{4 \lambda}{3 \sqrt{\pi}} \tau^{3 / 2}\right) \sqrt{t-\tau} d \tau=\frac{8 \lambda^{2}}{3 \pi} \int_{0}^{t} \tau^{3 / 2} \sqrt{t-\tau} d \tau=\frac{\lambda^{2} t^{3}}{3 !} .
\end{aligned}
$$


The first step of the double induction principle is just verified by (3.25) taking into account (3.30), (3.31). For the second step, we suppose by induction hypothesis that we have

$$
J_{2 n}(t)=\frac{\lambda^{2 n}}{(3 n) !} t^{3 n}, \quad J_{2 n+1}(t)=-\frac{2^{3 n+2}}{(3(2 n+1)) ! !} \frac{\lambda^{2 n+1}}{\sqrt{\pi}} t^{\frac{3(2 n+1)}{2}} .
$$

Therefore, we obtain

$$
\begin{aligned}
J_{2 n+2}(t) & =-\frac{2 \lambda}{\sqrt{\pi}} \int_{0}^{t} y_{2 n+1}(\tau) \sqrt{t-\tau} d \tau=\frac{\lambda^{2 n+2}}{\pi} \frac{2^{3 n+3}}{(6 n+3) ! !} \int_{0}^{t} \tau^{\frac{3(2 n+1)}{2}} \sqrt{t-\tau} d \tau \\
& =\frac{\lambda^{2 n+2}}{\pi} \frac{2^{3(n+1)}}{(6 n+3) ! !} \frac{\pi}{2^{3(n+1)}} \frac{(6 n+3) ! !}{(3(n+1)) !} t^{3(n+1)} \\
& =\frac{\lambda^{2 n+2}}{(3(n+1)) !} t^{3(n+1)}
\end{aligned}
$$

and

$$
\begin{aligned}
Y_{2 n+3}(t) & =-\frac{2 \lambda}{\sqrt{\pi}} \int_{0}^{t} y_{2 n+2}(\tau) \sqrt{t-\tau} d \tau=-\frac{2 \lambda^{2 n+3}}{(3(n+1)) ! \sqrt{\pi}} \int_{0}^{t} \tau^{3 n+3} \sqrt{t-\tau} d \tau \\
& =-\frac{2 \lambda^{2 n+3}}{(3(n+1)) ! \sqrt{\pi}} \frac{2^{3 n+4}(3(n+1)) !}{(6 n+9) ! !} t^{\frac{3(2 n+3)}{2}} \\
& =-\frac{2^{3(n+1)+2} \lambda^{2(n+1)+1}}{\sqrt{\pi}(3(2 n+1)+1) ! !} t^{\frac{3(n+1)+1}{2}} .
\end{aligned}
$$

This ends the proof.

Remark 3.6 Taking $t \rightarrow 0^{+}$in (3.14), (3.12), and (3.11), we obtain

$$
\begin{aligned}
& W\left(0^{+}\right)=+\infty, \quad W^{\prime}\left(0^{+}\right)=-\infty, \\
& U\left(0^{+}\right)=0, \quad U^{\prime}\left(0^{+}\right)=+\infty, \\
& g\left(0^{+}\right)=1, \quad g^{\prime}\left(0^{+}\right)=0 .
\end{aligned}
$$

So we deduce that the heat flux $W$ and the total heat flux $U$, and also $g$, are positive functions in a neighborhood of $t=0$.

\section{Conclusion}

We have obtained the global solution of a non-classical heat conduction problem in a semi- $n$-dimensional space. Moreover, for the one-dimensional case, we have obtained the explicit solution by using the Adomian method with a double induction principle.

\section{Competing interests}

The authors declare that they have no competing interests.

\section{Authors' contributions}

The authors declare that the work was realized in collaboration with the same responsibility. All authors read and approved the final manuscript.

\section{Author details}

'Institut Camille Jordan CNRS UMR 5208, Lyon University, 23 rue Paul Michelon 42023 Saint-Etienne Cedex 2,

Saint-Etienne, 42023, France. ${ }^{2}$ Departamento de Matemática-CONICET, FCE, Universidad Austral, Paraguay 1950, Rosario, S2000FZF, Argentina. 


\section{Acknowledgements}

This paper was partially sponsored by the Institut Camille Jordan St-Etienne University for first author, and the projects PIP \# 0534 from CONICET-Austral (Rosario, Argentina) and Grant AFOSR-SOARD FA 9550-14-1-0122 for the second author.

\section{Publisher's Note}

Springer Nature remains neutral with regard to jurisdictional claims in published maps and institutional affiliations.

Received: 5 October 2016 Accepted: 31 March 2017 Published online: 13 April 2017

\section{References}

1. Cannon, JR: The One-Dimensional Heat Equation. Addison-Wesley, Menlo Park (1984)

2. Carslaw, HS, Jaeger, CJ: Conduction of Heat in Solids. Clarendon, Oxford (1959)

3. Boukrouche, M, Tarzia, DA: Global solution to a non-classical heat problem in the semi-space $\mathbb{R}^{+} \times \mathbb{R}^{n-1}$. Q. Appl. Math. 72, 347-361 (2014)

4. Berrone, LR, Tarzia, DA, Villa, LT: Asymptotic behavior of a non-classical heat conduction problem for a semi-infinite material. Math. Methods Appl. Sci. 23, 1161-1177 (2000)

5. Ceretani, AN, Tarzia, DA, Villa, LT: Explicit solutions for a non-classical heat condition problem for a semi-infinite strip with a non-uniform heat source. Bound. Value Probl. 2015, 156 (2015)

6. Tarzia, DA, Villa, LT: Some nonlinear heat conduction problems for a semi-infinite strip with a non-uniform heat source. Rev. Unión Mat. Argent. 41, 99-114 (1998)

7. Villa, LT: Problemas de control para una ecuación unidimensional no homogénea del calor. Rev. Unión Mat. Argent. 32, 163-169 (1986)

8. Briozzo, AC, Tarzia, DA: Existence and uniqueness for one-phase Stefan problem of a non-classical heat equation with temperature boundary condition at a fixed face. Electron. J. Differ. Equ. 21, 16 (2006)

9. Briozzo, AC, Tarzia, DA: Exact solutions for nonclassical Stefan problems. Int. J. Differ. Equ. 2010, Article ID 868059 (2010)

10. Briozzo, AC, Tarzia, DA: A Stefan problem for a non-classical heat equation with a convective condition. Appl. Math Comput. 217, 4051-4060 (2010)

11. Salva, NN, Tarzia, DA, Villa, LT: An initial-boundary value problem for the one-dimensional non-classical heat equation in a slab. Bound. Value Probl. 2011, 4 (2011)

12. Cannon, JR, Yin, HM: A class of non-linear non-classical parabolic equations. J. Differ. Equ. 79, 266-288 (1989)

13. Glashoff, K, Sprekels, J: An application of Glicksberg's theorem to set-valued integral equations arising in the theory of thermostats. SIAM J. Math. Anal. 12, 477-486 (1981)

14. Glashoff, K, Sprekels, J: The regulation of temperature by thermostats and set-valued integral equations. J. Integral Equ. 4, 95-112 (1982)

15. Kenmochi, N: Heat conduction with a class of automatic heat source controls. Pitman Res. Notes Math. Ser. 186 471-474 (1990)

16. Kenmochi, N, Primicerio, M: One-dimensional heat conduction with a class of automatic heat source controls. IMA J. Appl. Math. 40, 205-216 (1988)

17. Bluman, G, Broadbridge, P, King, JR, Ward, MJ: Similarity: generalizations, applications and open problems. J. Eng. Math. 66, 1-9 (2010)

18. Friedman, A: Partial Differential Equations of Parabolic Type. Prentice Hall, New York (1964)

19. Ladyzenskaja, OA, Solonnikov, VA, Uralceva, NN: Linear and Quasilinear Equations of Parabolic Type. Am. Math. Soc., Providence (1968)

20. Miller, RK: Nonlinear Volterra Integral Equations. Benjamin, Elmsford (1971)

21. Bellman, R, Cooke, LL: Differential Difference Equations. Academic Press, New York (1963)

22. Adomian, G: Solving Frontier Problems of Physics Decomposition Method. Springer, Berlin (1994)

23. Adomian, G, Rach, R: Analytic solution of nonlinear boundary-value problems in several dimensions by decomposition. J. Math. Anal. Appl. 174, 118-137 (1993)

24. Wazwaz, AM: Linear and Nonlinear Integral Equations. Methods and Applications. Springer, Heidelberg (2011)

25. Adomian, G, Rach, R: Modified decomposition solution of linear and nonlinear boundary-value problems. Nonlinear Anal. TMA 23(5), 615-619 (1994)

26. Bougoffa, L, Mennouni, A, Rach, RC: Solving Cauchy integral equation of the first king by the Adomian decomposition method. Appl. Math. Comput. 219, 4423-4433 (2013)

27. Chen, W, Lu, Z: An algorithm for Adomian decomposition method. Appl. Math. Comput. 159, $221-235$ (2004)

28. Hosseini, MM, Nasabzadeh, H: On the convergence of Adomian decomposition method. Appl. Math. Comput. 182, 536-543 (2006)

29. Siddiqui, AM, Hameed, M: Adomian decomposition method applied to study nonlinear equations arising in non-Newtonian flows. Appl. Math. Sci. 6(98)، 4889-4909 (2012)

30. Wazwaz, AM: A comparison between Adomian decomposition method and Taylor series method in the series solutions. Appl. Math. Comput. 97, 37-44 (1998)

31. Wazwaz, AM, El-Sayad, SM: A new modification of the Adomian decomposition method for linear and nonlinear operators. Appl. Math. Comput. 122, 393-405 (2001)

32. Wazwaz, AM, Rach, R, Duan, JS: Adomian decomposition method for solving the Volterra integral form of the Lane-Emden equations with initial values and boundary conditions. Appl. Math. Comput. 219, 5004-5019 (2013)

33. Zhang, BQ, Luo, XG, Wu, QB: The restrictions and improvement of the Adomian decomposition method. Appl. Math. Comput. 177, 99-104 (2006) 